\title{
1 Organisatorische Voraussetzungen, Standard und medikolegale Aspekte
}

\author{
Frank Dette und Jürgen Graf
}

In der präklinischen Notfallmedizin sowie der Anästhesie und Intensivmedizin stellen Patienten mit ungewöhnlichen Körpermaßen, -formen oder -proportionen eine besondere Herausforderung dar. Hiervon sind gleichermaßen logistische Aspekte wie der präklinische und klinische Transport, aber auch diagnostische Maßnahmen und therapeutische Interventionen betroffen. Die Spanne der im Krankenhaus behandelten Individuen reicht von knapp $300 \mathrm{~g}$ leichten Frühgeborenen (im Göttinger Klinikum 2010) bis zum „schwersten Mensch der Welt", dessen Körpergewicht mit etwa 560 kg angegeben wurde. Ist die Auflagefläche des Schallkopfes des Sonografiegerätes für das Frühgeborene zu groß, so reicht die Eindringtiefe des Ultraschalls für den massiv Übergewichtigen für eine diagnostische Aussage nicht aus. Sind hier Zugangswege aufgrund haarfeiner Gefäße kaum zu etablieren, verdeckt auf der anderen Seite massives Fettgewebe die vorhandenen Venen.

Ausrüstung und Schulung der präklinischen und klinischen Versorgungsbereiche berücksichtigen naturgemäß den Standard und nicht die Extreme. Andererseits werden die Extreme im Hinblick auf gestiegene Körpermassen des Individuums in den Industriegesellschaften zunehmend häufiger (vgl. Kap.l.1). Dies beinhaltet nicht nur besondere Risiken und Komplikationen für die Fettleibigen selbst, sondern birgt auch Gefahren für die mit der Versorgung dieser Patienten betrauten nicht-ärztlichen und ärztlichen Mitarbeiterinnen und Mitarbeiter. Insofern erscheint es notwendig, dass sich Arbeitsbereiche mit einem hohen Anteil an notfallmedizinischen Patienten bereits im Vorfeld gezielt auf den Umgang bzw. die Betreuung von massiv adipösen Patienten vorbereiten, um im Notfall nicht unnötige Zeit zu verlieren oder die Patienten und sich selbst Gefahren auszusetzen.

\subsection{Rechtlicher Rahmen - medikolegale Aspekte}

Gesetzlich gibt es keinen Unterschied zwischen den $\mathrm{zu}$ betreuenden Individuen in Bezug auf die Körpermasse. Maßgeblich bleiben die im Sozialgesetzbuch V formulierten Ziele der Qualität der Patientenversorgung. Versicherungsrechtlich sollte der verantwortliche Arzt im Aufklärungsgespräch ein durch besondere anatomische Verhältnisse erhöhtes Interventionsrisiko - und damit möglicherweise verbundene Komplikationen - klar benennen und dieses auch im Aufklärungsbogen schriftlich fixieren. Bei der Anästhesieaufklärung gilt dies auch für den Ausschluss eines für den geplanten Eingriff sinnvollen oder üblicherweise eingesetzten Narkoseverfahrens, welches technische Schwierigkeiten bedingt durch anatomische oder physiologische Besonderheiten, aufweisen kann (Allgemeinanästhesie bei Eingriffen an der unteren Extremität, da Spinalanästhesie wegen unzureichender Nadellänge nicht durchführbar).

\subsubsection{Medizinprodukte}

Als bindend für den bestimmungsgemäßen Einsatz von medizinischen Gerätschaften ist das Medizinpro- 
duktegesetz (MPG) anzusehen. In der MedizinprodukteBetreiberverordnung (MPBetreibV) sind die Betreiber und Anwendervorschriften entsprechend definiert. Finden sich hier Gewichtsgrenzwerte für die Tragfähigkeit von z.B. Betten, Krankentragen, Operationstischen oder auch Röntgenanlagen, so sind diese unbedingt auch im Einzelfall einzuhalten. Schäden, die aus Nichtbeachtung von gerätespezifischen Grenzwerten resultieren, können im Einzelfall als grob fahrlässig betrachtet werden und somit Schadensersatzforderungen nach sich ziehen.

Ähnliches gilt für prophylaktische Maßnahmen: Ist durch anatomische Gegebenheiten mit einem schwierigen Atemweg zu rechnen oder erscheint eine Aspiration aufgrund einer massiven Adipositas wahrscheinlich, so sind diese Risiken in die Verfahrensplanung mit aufzunehmen.

Analog stellt sich die Notwendigkeit spezieller Betten mit Wechseldruckmatratzensystemen oder auch eine entsprechend angepasste Lagerung bei operativen Eingriffen mit dem Ziel der Vermeidung von Druckulzerationen. Die angepasste, korrekte Lagerung sowie der Hautbefund sollte regelmäßig Eingang in die Dokumentation finden - im Operationssaal genauso wie auf der Überwachungs- oder Normalstation.

\subsubsection{Arbeitsschutz}

Neben einem erhöhten Risiko der schwergewichtigen Patienten sind auch die betreuenden Mitarbeiter spezifischen Risiken ausgesetzt. Fürsorgepflichtig ist hier der Arbeitgeber, unterstützt werden Arbeitgeber in der Wahrnehmung dieser Fürsorge durch die Arbeits- bzw. Betriebsmediziner, die Arbeitssicherheit oder Fachkraft für Arbeitssicherheit und die Berufsgenossenschaften. Hier können Arbeitnehmer und Arbeitgeber auch Informationen hinsichtlich des notwendigen oder empfehlenswerten Arbeits-bzw. Gesundheitsschutzes erhalten.

Im Zusammenhang mit fettleibigen Patienten entstehen die wesentlichen Risiken für die Mitarbeiter durch die Gewichtsbelastung beim Heben und Tragen. Sowohl präklinisch als auch im stationären Bereich muss oft durch medizinisches Personal im Rahmen von Transporten oder notwendigen Umlagerungen das Körpergewicht des Patienten gegen die Schwerkraft bewegt werden. Hieraus resultierende Schädigungen, die zur Aufgabe der Tätigkeit zwingen, können in Einzelfällen als Berufskrankheit anerkannt werden (BK Nummer 2108 und 2109, Anlage 1 zur Berufskrankheiten-Verordnung, Bundesgesetz- blatt I, Seite 1273, Sozialgesetzbuch VII). Wo immer möglich, sollten den Mitarbeitern geeignete technische oder organisatorische Hilfen zur gesundheitsverträglichen Bewältigung der Last zur Verfügung stehen. Dies können Hebehilfen (Lifter) und Lagerungsunterstützungen (Rollboards) sein, aber auch Universalliegen, die für Transport und Diagnostik ohne Umlagerung gleichermaßen geeignet sind.

Vergleichsweise seltener besteht das Risiko einer im Vergleich zum Individuum mit Normalmaß erhöhten Strahlenexposition aufgrund der höheren Energiedosis und vermehrter Streustrahlung. Dennoch ist bei Durchleuchtungen (z.B. im Rahmen einer ERCP oder Herzkatheteruntersuchung) auf alle Möglichkeiten des Strahlenschutzes zu achten: Neben möglichst kurzen Durchleuchtungszeiten sowie der Ausnutzung des maximal möglichen Abstandes zur Strahlenquelle zählen hierzu auch verbleite Abschirmungen und Bleiglasstellwände, Bleischürzen und Bleiglasbrillen sowie eine strenge Indikationsstellung (individuelle Risiko-Nutzen-Abwägung).

\subsection{Organisatorische Voraussetzungen und Standards}

Eine aus organisatorischer Sicht im Krankenhaus wesentliche Differenzierung unterscheidet geplante und entsprechend vorbereitete Krankenhausaufnahmen von ungeplanten, häufig notfallmäßigen Hospitalisationen. Die besondere Herausforderung des stationären Versorgungssystems besteht darin, den elektiven Regelbetrieb unter der Berücksichtigung der vorhandenen Ressourcen nicht durch die ungeplanten Krankenhausaufnahmen zu beeinträchtigen. Andererseits stellen auch ungeplante Aufnahmen - je nach Versorgungsauftrag - einen wichtigen Deckungsbeitrag für die medizinischen Einrichtungen dar. Idealerweise lässt sich dies mit nur geringen Überkapazitäten ohne kritische Engpässe bewerkstelligen. Dies ermöglicht dem Krankenhausbetreiber - je nach Ausrichtung - ein hohes Maß an Patienten- und Mitarbeiterzufriedenheit durch schlanke Prozesse und schafft die theoretische Grundlage für eine medizinisch und wirtschaftlich erfolgreiche Arbeit.

Es erscheint somit für jedes Krankenhaus sinnvoll, die eigenen Patientenkollektive, Patientenströme sowie die für die Versorgung notwendigen Strukturen und Prozesse sorgfältig zu analysieren, um Defizite in der Planung, den Diagnose-, Therapie- sowie Interventionsleistungen und bei der Ressourcennutzung zu vermeiden. 
Verschiedene Patientengruppen stellen allerdings trotz sorgfältiger Analyse und intelligenter Planungssysteme immer noch eine medizinische wie auch organisatorische Herausforderung im Klinikalltag dar. Hierzu gehören zweifelsohne die stark übergewichtigen Patienten und Patienten mit ungewöhnlicher Körperfülle bzw. Verteilung der Körpermasse (The Association of Anaesthesists 2007).

\subsubsection{Patiententransport - Prähospitale Transportmodalitäten}

Im präklinischen Transport sehen sich die Rettungsdienste oder medizinischen Transportdienstleister oftmals noch größeren Herausforderungen gegenüber, als dies im Innerhospital- oder Interhospitaltransfer üblich ist: Private Wohnungen und Häuser weisen häufig schmale und steile Treppenhäuser auf und besitzen keinen Lift, sodass der Transport nur mit den vorhandenen Tragehilfen (Tragestuhl oder Krankentrage, gegebenenfalls Schaufeltrage) gewährleistet werden muss. Um gefährliche Hebe- und Tragesituationen zu vermeiden und den Arbeitsschutz für das Personal zu gewährleisten, müssen für den präklinischen Transport von schwergewichtigen Patienten im Vorfeld klare Regelungen getroffen werden, die idealerweise als „Standard operatingprocedure“ (SOP) allen Mitarbeitern und Schnittstellenpartnern bekannt sind.

Hier sind eine Reihe von Szenarien denkbar, beginnend mit dem Anfordern einer zweiten Rettungswagenbesatzung bis hin zur Einbindung der Feuerwehr zum Transport eines Patienten über Leiter oder Korb außerhalb des Hauses. Derartige Transportmodalitäten werden nur dann in der Praxis mit der notwendigen Geschwindigkeit zur Verfügung stehen, wenn alle beteiligten Systempartner diese Szenarien miteinander vereinbart und als SOP schriftlich fixiert haben (s. Tab. 1). Idealerweise werden auch gemeinsame Simulationen oder sogar Übungen durchgeführt.

Eine augenscheinliche Limitation beim Transport schwer übergewichtiger Patienten stellt die Fahrtrage dar, die aufgrund der Bestimmungen des MPG bzw. der MPBetreibV für den Transport eines entsprechenden schwergewichtigen Patienten ausgelegt sein muss. Die für Krankentransportmittel im Krankenkraftwagen gültige Deutsche Industrie-Norm (DIN) EN 1865-5 (Ausgabe 03-2010) fordert eine maximale Belastbarkeit der eingesetzten Tragen von $300 \mathrm{~kg}$ Körpergewicht (Deutsches Institut für Normung 2010). Ein Transport von Patienten mit einem Kör-
Tab. 1 Checkliste zu Rettung und präklinischem Transport schwergewichtiger Patienten

\section{Ja Nein}

1. Kann der Patient mit dem vorhandenen Material (Fahrzeug, Trage, Gurtsysteme, Monitoring) sicher transportiert werden?

2. Ist zum Transport bzw. zur Rettung des Patienten ausreichend Personal vorhanden?

3. Sind die Transport- bzw. Rettungswege geeignet (Breite? Steigung und Radien von Flur bzw. Treppenhaus?)

4. Existiert eine sichere Transportalternative (z.B. Außentransport mittels FeuerwehrDrehleiter oder Kran)?

pergewicht über $300 \mathrm{~kg}$ ist somit mit den üblichen Krankenfahrtragen nicht möglich und erfordert den Einsatz eines Spezialfahrzeugs mit entsprechender Ausrüstung (s. Abb. 1). Unabhängig davon ist zu beachten, dass längst nicht alle Transportmittel die DIN von 2010 erfüllen, d.h. auch unterhalb von $300 \mathrm{~kg}$ nicht jederzeit (rechts-)sicher eingesetzt werden können.

Neben der Belastbarkeit des Transportmittels sind auch die Breite der Krankentrage sowie adäquate Gurt- bzw. Sicherheitssysteme zur Sicherung des Patienten und Fixierung der Fahrtrage im Fahrzeug sowie Haltegriffe für den Transport von wesentlicher Bedeutung. Sowohl für den Transfer auf die Krankentrage, als auch für den Transport zum und in das Rettungsfahrzeug sind entsprechende Hilfsmittel sowie geeignete und geschulte Mitarbeiter in ausreichender Zahl notwendig.

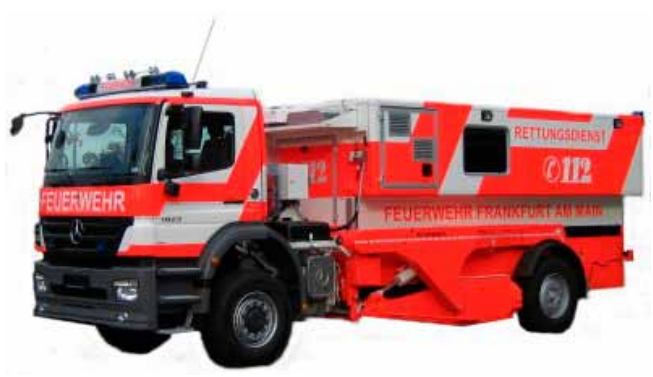

Abb. 1 Patienten-Schwerlasttransporter der Berufsfeuerwehr Frankfurt am Main (mit freundlicher Genehmigung der Berufsfeuerwehr Frankfurt am Main) 
Die Berufsgenossenschaften empfehlen bei gelegentlichem Heben und Tragen eine Last von etwa $50 \mathrm{~kg}$ für männliche und $15 \mathrm{~kg}$ für weibliche Mitarbeiter nicht zu überschreiten (s. Tab. 2). Dies gilt bei optimierter Rückenhaltung.

Demzufolge müssten für einen Patienten mit 250 kg Körpergewicht mindestens 5 männliche Personen für Transfer und Abtransport zur Verfügung stehen.

Luftgebundene Rettungs- und Transportmittel (Rettungshubschrauber, Intensivtransporte, Ambulanzflüge, Linienflüge und ähnliches) sind oftmals in besonderem Maße sowohl Raumbeschränkungen als auch Gewichtslimitationen und gesetzlichen bzw. flugbetrieblichen Regularien unterworfen. Dies ist im Vorfeld aufgrund der Vielfalt der eingesetzten Flugmuster und Ausstattungsalternativen unbedingt individuell abzuklären.

\subsubsection{Patiententransport - Intrahospitale Transportmodalitäten}

Die vorgenannten Anforderungen an medizinische Geräte und Einrichtungsgegenstände gemäß MPG sind analog auf alle in der stationären Versorgung (Spezialbetten) und im innerklinischen Transport sowie Patiententransfer eingesetzten Produkte (Tragen, Schleusen, Operationstische, Deckenlifter und vieles mehr) anwendbar(s. Tab. 3). Gewöhnliche Operationstische sind laut Herstellerangaben für eine maximale Tragfähigkeit von etwa $250 \mathrm{~kg}$, vereinzelt auch bis $360 \mathrm{~kg}$, ausgelegt. Bei Patienten mit darüber hinausgehendem Körpergewicht müssen spezielle Schwerlast-Operationstische mit ausreichender Breite und entsprechenden Lagerungsmöglichkeiten zum Einsatz kommen. Diese sind - je nach Hersteller - teilweise bis $500 \mathrm{~kg}$ belastbar. Hinsichtlich des bei Transfer und Lagerung benötigten Personals sollten auch intrahospital die Grenzwerte aus Tabelle 2 Anwendung finden. Zur Erinnerung: Die Umlagerung eines $150 \mathrm{~kg}$ schweren Patienten hat - im Falle der Verfügbarkeit nur weiblicher Mitarbeiterinnen, durch 10 Personen zu erfolgen.

Die übliche Belastungsgrenze von Deckenliften und Hebehilfen liegt je nach Modell zwischen $200 \mathrm{~kg}$ und $300 \mathrm{~kg}$, wobei hier die statischen Voraussetzungen der Gebäudedecke bzw. die maximale Last pro Quadrameter Fläche für z.B. Neuausstattungen ebenfalls zu beachten sind.

Im klinischen Alltag werden mangels adäquater Vorbereitung, Unwissenheit oder fehlender Ausstattung immer wieder für Patienten und Mitarbeiter
Tab. 2 Orientierungshilfe zur Zumutbarkeit von Lasten beim Heben und Tragen (Bundesministerium für Arbeit und Soziales 1981)

\begin{tabular}{l|cc|cc} 
& \multicolumn{3}{c}{ Zumutbare Last in kg } \\
& \multicolumn{2}{c}{ Häufigkeit des Hebens und Tragens } \\
\hline & \multicolumn{2}{c}{ gelegentlich } & \multicolumn{2}{c}{ häufiger } \\
\hline Lebensalter & Frauen & Männer & Frauen & Männer \\
\hline 15 bis 18 Jahre & 15 & 35 & 10 & 20 \\
\hline 19 bis 45 Jahre & 15 & 55 & 10 & 30 \\
\hline $\begin{array}{l}\text { älter als } \\
\text { 45 Jahre }\end{array}$ & 15 & 45 & 10 & 25 \\
\hline
\end{tabular}

Gelegentlich: Heben und Tragen der Last höchstens 1 x pro Stunde bei einem Transportweg bis längstens 4 Schritte

Häufiger: Heben und Tragen der Last wenigstens $2 \times$ pro Stunde bei einem Transportweg von 5 und mehr Schritten

Tab. 3 Checkliste zu innerklinischem Transport schwergewichtiger Patienten, Belastungsgrenzen diagnostischer und interventioneller Geräte

1. Stehen Tragen und Betten mit der notwendigen Breite, Tragfähigkeit sowie Wechseldruck-Matratzensysteme zur Verfügung?

2. Sind Wege und Türen auch für Spezialbetten oder -tragen breit genug (auch z.B. im OP-Bereich, der Radiologie, dem Herzkatheter?)

3. Sind Hebe- und Lagerungseinrichtungen (Lifter) vorhanden und steht geeignetes Gerät zur Umlagerung (Rollbretter u.ä.) zur Verfügung?

4. Ist genügend Personal unter Berücksichtigung der Maximallast des Einzelnen für den Transport verfügbar?

5. Sind die diagnostischen und interventionellen Geräte (z.B. CT-Liege) für diese Gewichtsbelastung ausgelegt/ zugelassen?

gleichermaßen inakzeptable Kompromisse eingegangen (z.B. Fixierung von Patienten auf deutlich zu schmalen Tragen mittels Klebeband, Nebeneinanderstellen von zwei OP-Tischen etc.). Dies schränkt die Sicherheit für alle Beteiligten in unzumutbarer 
Weise ein und sollte nicht zuletzt aus versicherungsrechtlicher Sicht nicht toleriert werden.

\subsubsection{Diagnostische und interventionelle Maßnahmen}

Punktionen von Nerven und Gefäßen gestalten sich bei adipösen Patienten mitunter schwierig. Neben geeignetem Material (adäquate Länge von Nadeln und Kathetern) und der zielgerichteten Lagerung können Alternativmethoden wie z.B. die intra-ossäre Punktion als Infusionszugang oder Ultraschall zur Identifikation der Zielgefäße eine sinnvolle Ergänzung darstellen. Neben dem Vorhandensein der notwendigen Materialien und Geräte ist allerdings auch Sorge zu tragen, dass die Mitarbeiter in den Umgang, die Techniken und die damit verbundenen allgemeinen Verfahrensweisen eingewiesen sind und diese ausreichend häufig durchführen - entweder im Rahmen von Simulationen, oder aber in der klinischen Anwendung, z.B. auch bei unproblematischen Patienten.

Für die Ultraschall-unterstützte Regionalanästhesie bei adipösen Patienten wurde im klinischen Setting eine den Normalgewichtigen vergleichbare Erfolgsrate erzielt (Schwemmer et al. 2006). Auch für die Durchführung neuroaxialer Verfahren scheint der Ultraschall eine sinnvolle Unterstützung darzustellen, wenn eine visuelle oder taktile Orientierung zum Aufsuchen der gewünschten Punktionsstelle aufgrund von Fettpolstern nicht möglich ist (Ecimovic u. Loughrey 2010; Stiffler et al. 2007).

Obwohl der Ultraschall eine sinnvolle Ergänzung zur Erhöhung der Erfolgsrate, z.B. bei perkutanen Zugangswegen, darstellen kann, leidet auch diese Methode unter den physikalischen Gesetzmäßigkeiten: Lange Vorlaufstrecken aufgrund massiven Unterhautfettgewebes schränken das Punkt-Auflösungsvermögen erheblich ein und können sogar dazu führen, dass mit den vorhandenen Schallköpfen eine bildgebende Diagnostik nicht durchführbar ist (s. Abb. 2).

Für die Computertomografie, Magnetresonanztomografie, Koronarangiografie und ähnliche Verfahren spielt die bereits erwähnte maximal zulässige Gewichtsbelastung der Geräteliegen sowie deren Länge, Breite, Lagerungs- und Fixierungsmöglichkeit eine Rolle. In geschlossenen Diagnostikeinheiten kann auch der jeweilige Geräteausschnitt oder die notwendige Spule (Kernspintomografie) einen limitierenden Faktor darstellen.

Neben diesen statisch-logistischen Schwierigkeiten besteht für alle diagnostischen Systeme eine

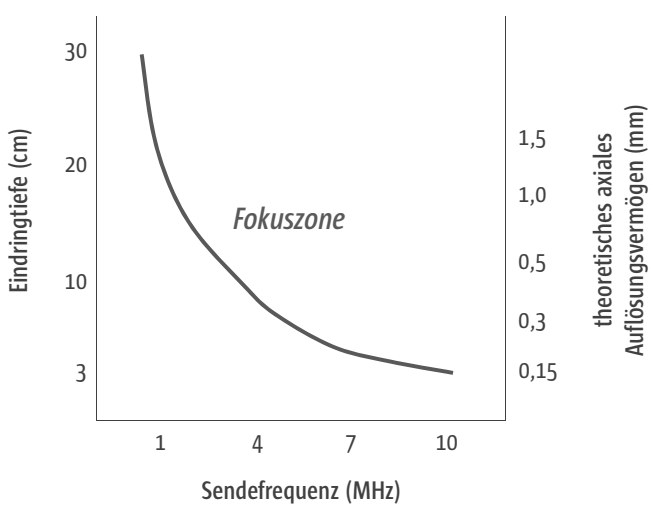

Abb. 2 Beziehung von Gewebeeindringtiefe, Sendefrequenz des Schallkopfes und theoretischem axialem Auflösungsvermögen

Abhängigkeit zwischen Energiedosis, Gewebeeindringtiefe und Auflösungsvermögen. Eine Computertomografie mit signifikant eingeschränkter Bildqualität kann Strahlendosen erreichen, die dem Doppelten der Dosis bei einem normalgewichtigen Kontrollkollektiv entsprechen (Bamberg et al. 2011). Neben der Auswahl der geeigneten diagnostischen Verfahren sollte deshalb auch die Indikation für den Einsatz der Verfahren streng gestellt werden, da der Nutzen u.U. hinter den sonst erzielbaren Ergebnissen deutlich zurückbleibt.

Selbst die traditionelle klinische Untersuchung (Inspektion und Palpation) und die Auskultation mittels Stethoskop sind bei massiv adipösen Patienten nur bedingt aussagekräftig, da Strukturen weder gesehen, noch ertastet werden können und die Eindringtiefe bzw. Schallübertragung mit dem Stethoskop auf 4-5 cm Gewebetiefe beschränkt ist. Bei fettleibigen Patienten wird der wahrnehmbare Schall bisweilen somit vollständig von Fettpolstern gedämpft.

\subsubsection{Lagerungsmaßnahmen}

Besondere Aufmerksamkeit muss im Rahmen der Versorgung adipöser Patienten auf eine konsequente Lagerung gerichtet werden. Fettgewebe ist kaum vaskularisiert und reagiert konsekutiv vulnerabler auf Druck (Gallagher et al. 2004). Andererseits werden gerade massiv übergewichtige Patienten immer wieder auch, z.B. bei Wirbelsäuleneingriffen, in die Bauchlage verbracht. Um Druckulcera zu vermeiden, müssen druckempfindliche Areale suffizient gepolstert werden. Darüber hinaus ist eine regelmäßige In- 
spektion erforderlich, um Schäden durch Umlagerung zu vermeiden (Vollmar et al. 2001).

Für die Lagerung gelten hinsichtlich der Mitarbeiterbelastung die bereits skizzierten Rahmenbedingungen, d.h. auch hier ist auf eine ausreichende Anzahl entsprechender Mitarbeiter für eine sichere und zielgerichtete Durchführung der Maßnahmen zu achten.

Überdies sind auf organisatorischem Weg Lagerungshilfen (Gelkissen, Wechseldruckmatratzen mit periodischer Druckentlastung, Schalen und Schienen) vorzuhalten. Neben Druck stellt Feuchtigkeit eine Hauptursache für lagerungsbedingte Hautläsionen dar. Feuchtigkeit, bei adipösen Patienten vermehrt auch in Hautfalten, bildet einen idealen Nährboden für Keime und bildet somit eine ideale Grundlage für Infektionen der Haut (Wilson u. Clark 2004). Aufgrund der besonderen Bedeutung - auch in Bezug auf Komplikationen mit zu erwartender Wundheilungsstörung - ist jede der durchgeführten Maßnahmen akribisch zu dokumentieren. Dies sollte als Checkliste gestaltet und im Verlauf länger andauernder Interventionen immer wieder kontrolliert und neu dokumentiert werden.

\subsubsection{Thromboseprophylaxe}

Adipöse Patienten haben ein signifikant erhöhtes Risiko für thrombembolische Ereignisse. Eine adäquate mechanische und pharmakologische Prophylaxe ist daher essenziell. Eine Kombination aus Stützstrümpfen und niedermolekularem Heparin wird mit einem Evidenz-Grad B empfohlen (Adams u. Murphy 200o). Einer aktuellen Metaanalyse zufolge kann auch bei adipösen Patienten das Körpergewicht als Dosierungsgrundlage des niedermolekularen Heparins herangezogen werden. Eine laborchemische Erfolgskontrolle (Anti-Xa-Spiegel) wird bei morbider Adipositas jedoch empfohlen (Nutescu et al. 2009). Für neuere Substanzen und die Gruppe der oralen Gerinnungshemmer liegen bislang keine Daten für die Anwendung bei massiv übergewichtigen Patienten vor.

\section{Zusammenfassung}

Um auch in Notfallsituationen einen akzeptablen Sicherheitsstandard für übergewichtige Patienten und für Mit- arbeiter gewährleisten zu können, wird die Etablierung von Protokollen bzw. SOPs (standard operating procedures) zur Versorgung von Patienten mit morbider Adipositas empfohlen (The Association of Anaesthesists 2007). Diese umfassen organisatorische Bereiche (Bereitstellung von speziellen Hilfsmitteln, Checklisten zu inner- oder intraklinischen Transporten, Überprüfung von baulichen und personellen Voraussetzungen) ebenso wie die sorgfältige Kenntnis spezifischer medizinischer Grundlagen (z.B. Gefäßpunktionen, Thromboseprophylaxe).

\section{Literatur}

Adams IP, Murphy PG (2000) Obesity in anaesthesia and intensive care. Br | Anaesth 85, 91-108

Bamberg F, Marcus R, Petersilka M, Nikolaou K, Becker CR, Reiser MF, Johnson T (2011) Herausforderungen an die Computertomographie bei übergewichtigen Patienten. Radiologe 51, 366-371

Bundesministerium für Arbeit und Soziales (Hrsg.) (1981) Bundesarbeitsblatt 11, 96

Deutsches Institut für Normung (2010) Krankentransportmittel im Krankenkraftwagen - Teil 5: Festlegungen zur Krankentragenaufnahme; Deutsche Fassung prEN 1865-5:2010

Ecimovic P, Loughrey JP (2010) Ultrasound in obstetric anaesthesia: a review of current applications. Int J Obstet Anesth 19 320-326

Gallagher S, Langlois C, Spacht DW, Blackett A, Henns T (2004) Preplanning with protocols for skin and wound care in obese patients. Adv Skin Wound Care 17, 436-441

Nutescu EA, Spinler SA, Wittkowsky A, Dager WE (2009) Low-molecular-weight heparins in renal impairment and obesity: available evidence and clinical practice recommendations across medical and surgical settings. Ann Pharmacother 43, 10641083

Schwemmer U, Papenfuss T, Greim C, Brederlau I, Roewer N (2006) Ultrasound-guided interscalene brachial plexus anaesthesia: differences in success between patients of normal and excessive weight. Ultraschall Med 27, 245-250

Stiffler KA, Jwayyed S, Wilber ST, Robinson A (2007) The use of ultrasound to identify pertinent landmarks for lumbar puncture. Am J Emerg Med 25, 331-334

The Association of Anaesthesists of Great Britain and Ireland (AAGBI) (2007) Peri-operative management of the morbidly obese patient, http://www.aagbi.org/sites/default/files/Obesity07.pdf, zugegriffen am 26.10.2011

Vollmar HC, Butzlaff M, Koneczny N, Floer B (2001) Leitlinie Dekubitusprävention, Version 11/2001 http://www.evidence.de/ Leitlinien/leitlinien-intern/Dekubitus_Leitlinie_Evidence_d/ dekubitus_leitlinie_evidence_d.html, zugegriffen am 26.10.2011

Wilson JA, Clark I| (2004) Obesity: impediment to postsurgical wound healing. Adv Skin Wound Care 17, 426-435 


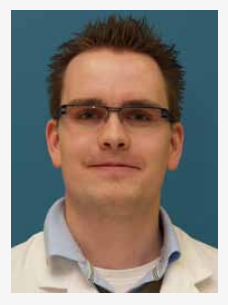

\section{Dr. med. Frank Gerhard Dette}

Studium der Humanmedizin an der Philipps-Universität Marburg, 2007 Staatsexamen. Seit 2008 wissenschaftlicher Mitarbeiter der Universitätsklinik für Anästhesie und Intensivtherapie an der Philipps-Universität Marburg. Wissenschaftlicher Schwerpunkt: Schlafbezogene Atmungsstörungen im perioperativen Umfeld.

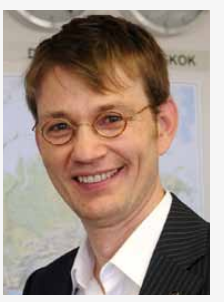

\section{Prof. Dr. med. Jürgen Graf}

Studium der Medizin an der RWTH Aachen. 2005 Facharzt für Innere Medizin, 2009 Facharzt Anästhesiologie und Intensivmedizin, Notarzt, Flug- und Reisemediziner. 2007 Habilitation im Fach Intensivmedizin, 2011 außerplanmäßige Professur an der Philipps-Universität Marburg. Wissenschaftliche Schwerpunkte: Qualitäts- und Risikomanagement, akute und chronische Herzinsuffizienz, Fibrose und Fibrolyse. Seit 2009 Leitender Arzt der Passenger Medical Care und Medical Operation Center des Medizinischen Dienstes der Deutschen Lufthansa AG. 